\title{
Temperature-dependent phase transformation of ice-1h under ultrafast uniaxial compression: A molecular dynamics simulation
}

\author{
Qiuyun Yin ${ }^{\mathrm{a}, *}$, Lingling $\mathrm{Hu}^{\mathrm{a}}$, Xianqian $\mathrm{Wu}^{\mathrm{b}, *}$, Kailu Xiao ${ }^{\mathrm{b}}$, Chenguang Huang ${ }^{\mathrm{b}}$ \\ ${ }^{a}$ Department of Applied Mechanics \& Engineering, School of Engineering, Sun Yat-sen University, Guangzhou 510275, PR China \\ ${ }^{\mathrm{b}}$ Key Laboratory of Mechanics in Fluid Solid Coupling Systems, Institute of Mechanics, Chinese Academy of Sciences, Beijing 100190, PR China
}

\section{A R T I C L E I N F O}

\section{Keywords:}

Ice

Phase transformation

Dynamic behaviour

Molecular dynamics

\begin{abstract}
A B S T R A C T
The temperature and strain rate-dependent compressive behaviour of ice- $1 \mathrm{~h}$ was investigated through molecular dynamics simulations. The ice strength increased in response to a decrease in the initial temperature and an increase in the strain rate. Various deformation mechanisms depended on the ice's initial temperature. Solidliquid phase transformation was more likely to at a relatively high temperature. However, solid-solid phase transformation and dislocation-like glide were observed at relatively low temperatures. Experimental observations on the strength versus strain rate relationship of ice could be interpreted based on a prior study by Wu and Prakash [1].
\end{abstract}

\section{Introduction}

The dynamic behaviour of ice plays a crucial role in diverse subjects such as glaciology [2], avalanches, icebreaking in the Arctic, and climatology. For instance, the prediction of the movement or breakup of glaciers, which can change the landform, requires knowledge of the dynamic behaviour of ice.

Many studies have focused on the mechanical behaviour of ice [3-13]. Schulson et al. [7] observed that the strength of ice increased with the increasing strain rate in a range from $10^{-2}$ to $1.6 \mathrm{~s}^{-1}$, consistent with the results of Jones [14] at a higher strain rate up to $10^{1} \mathrm{~s}^{-1}$ and a temperature of $-11^{\circ} \mathrm{C}$. Moreover, Jones [14] showed that the strength of freshwater ice was higher than sea ice at a relatively low strain rate. Kim et al. [15] studied the dynamic behaviour of ice through the split Hopkinson pressure bar (SHPB) in a strain rate ranging from 400 to $2600 \mathrm{~s}^{-1}$. They concluded that the ice compression strength was constant at $19.7 \mathrm{MPa}$ in the test strain rate range. The high strain rate behaviour of ice was also tested by Shazly and Prakash [16] through SHPB. Their results showed that the compressive strength of ice exhibited positive strain rate sensitivity over the strain rates ranging from 60 to $1400 \mathrm{~s}^{-1}$. A similar conclusion was derived by Wu and Prakash $[1,17]$. Wu and Prakash [1] reported that the peak compressive strength demonstrated two different types of temperature sensitivity, that is, the strength increased from 32 to $112 \mathrm{MPa}$ as the test temperature decreased from $-15^{\circ} \mathrm{C}$ to $-125^{\circ} \mathrm{C}$, followed by a nearly constant value in a range of 110-120 MPa with a further decrease in the temperature to $-173^{\circ} \mathrm{C}$. Although Wu and Prakash [1] speculated that phase transformation might occur during ice compression at ultralow temperatures, the actual cause remains unknown. Further studies are required to understand the dynamic compressive behaviour of ice at various ambient temperatures.

A variety of microstructures, that is, 13 crystal structures and two amorphous states, that have been discovered to date depending on the growth temperatures and pressure conditions cause the complexity of mechanical behaviour of ice as previously noted. The study of microstructural evolution during compression provides a pathway to better understand the properties of ice. Many molecular dynamics (MD) simulations have been performed to study the properties of ice [18-27]. Karim et al. [19] reported via MD simulations using the TIP4P model of water that the interface of ice/water was stable at temperatures of $240 \pm 1.5 \mathrm{~K}$. Gay et al. [28] studied the melting process and stability of ice using the SPCE model of water. The results showed that ice-1h structures at 1 atmosphere were almost identical as the temperature increased close to the melting transition point. Matsumoto et al. [29] investigated ice nucleation and growth behaviour. The results demonstrated that the nucleation of freezing necessitates sufficient long-lived hydrogen bonds developing spontaneously at the same location before a rapid expansion. Despite numerous MD simulations of the properties of ice and its freezing/melting mechanics, to the best of our knowledge, studies on the initiation of complex dynamic behaviour of ice that are required to better understand the phenomenon observed by Wu et al. [1] remain limited.

\footnotetext{
* Corresponding authors.

E-mail addresses: yinqy5@mail.sysu.edu.cn (Q. Yin), wuxianqian@imech.ac.cn (X. Wu).
} 


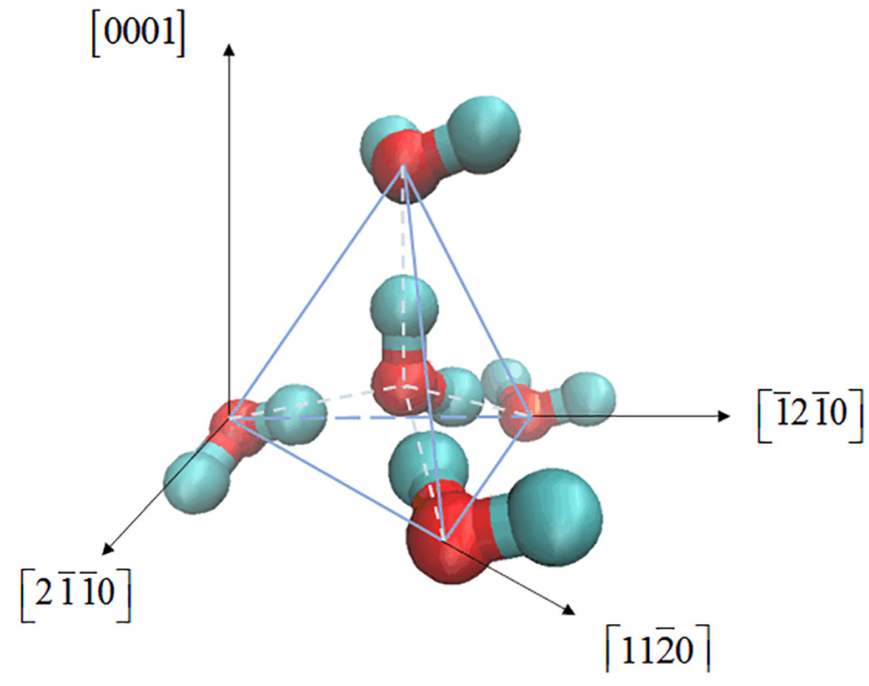

Fig. 1. Structure of a single crystal cell of ice- $1 \mathrm{~h}$.

With this as a motivation, MD simulations focused on the phase transformation of ice during high strain rate loading were performed. This study investigated the temperature and strain rate effects on the

(a)

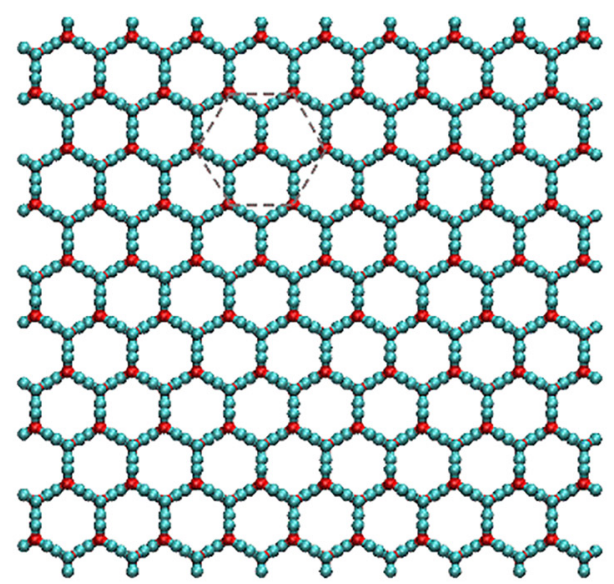

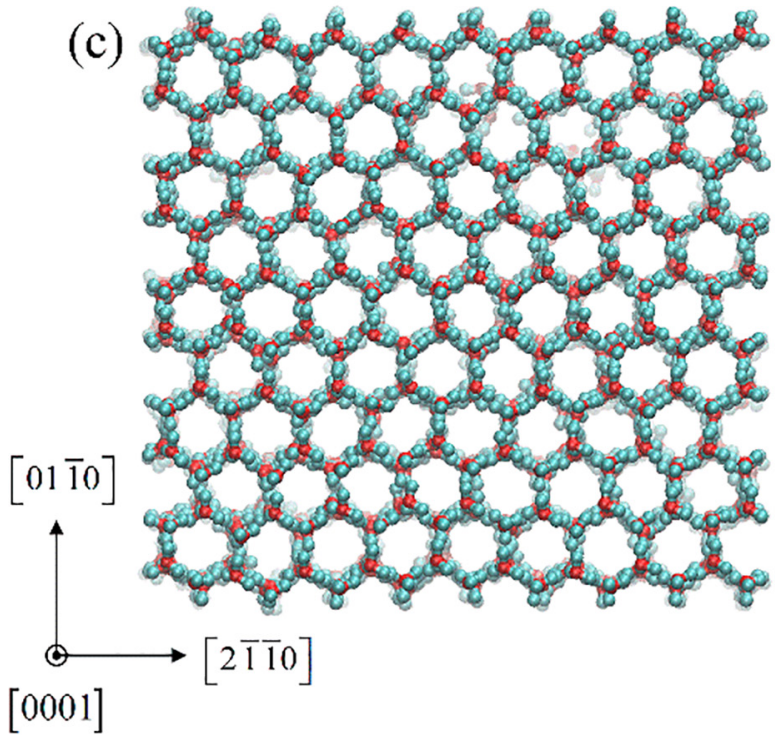

Fig. 2. Ice structures of the simulation model. (a) Before relaxing from [ $\left[\begin{array}{llll}0 & 0 & 0 & 1\end{array}\right]$. (b) Before relaxing from [ $\left.\begin{array}{llll}2 & \overline{1} & 0\end{array}\right]$. (c) After relaxing at $263 \mathrm{~K}$ from [0 00 l]. (d) After relaxing at $263 \mathrm{~K}$ from [ $\left[\begin{array}{llll}2 & \overline{1} & 0 & 0\end{array}\right]$. strength and the deformation mechanisms of ice.

This paper is organised as follows. The atomistic models of ice and the details of the simulation method are described in Section 2. The phase transformation, temperature effect on the strength of ice, and temperature-crystal structure interactions during compression are discussed in Section 3. The results are summarised in Section 4.

\section{Methods}

Large-scale atom/molecular massively parallel simulation (LAMMPS) [30], a widely used open-source software, was used to perform the MD simulations. Fig. 1 shows a single crystal cell of ice, where a 4-index notation for the hexagonal system is adopted for the coordinate axes. As demonstrated in Fig. 1, a water molecular was constructed using one oxygen atom (red sphere) and two hydrogen atoms (blue sphere). The bond length between the oxygen-hydrogen atoms is $0.99 \AA$ and the angle of the hydrogen-oxygen-hydrogen atoms is $104^{\circ}$. The ice structure is adopted by ice- $1 \mathrm{~h}$ [26], that is, there are four neighbouring oxygen atoms around one oxygen atom to construct a regular tetrahedron.

Many potentials such as SPCE [28], TIP3P [31], and TIP4P [32] have been developed to describe the interaction of water molecules. SPCE was used in this study because of its high efficiency and ability to describe the properties of bulk water $[28,33]$.

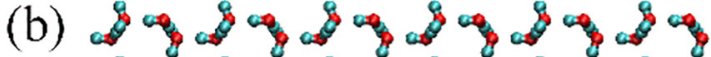

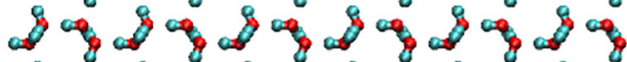

$$
\begin{aligned}
& \text { \& o o o o o }
\end{aligned}
$$

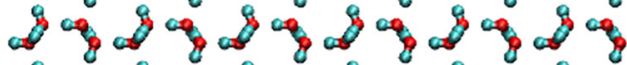

$$
\begin{aligned}
& \text { \& \% } 80 \% \\
& \text { \& } 80 \text { \% } 808
\end{aligned}
$$

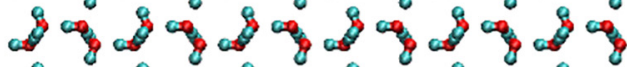

$$
\begin{aligned}
& \text { \& \& } 80 \% \\
& \text { \& } 8 \text { \% } 8
\end{aligned}
$$

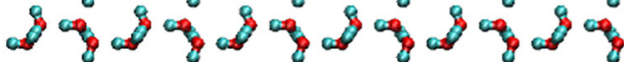

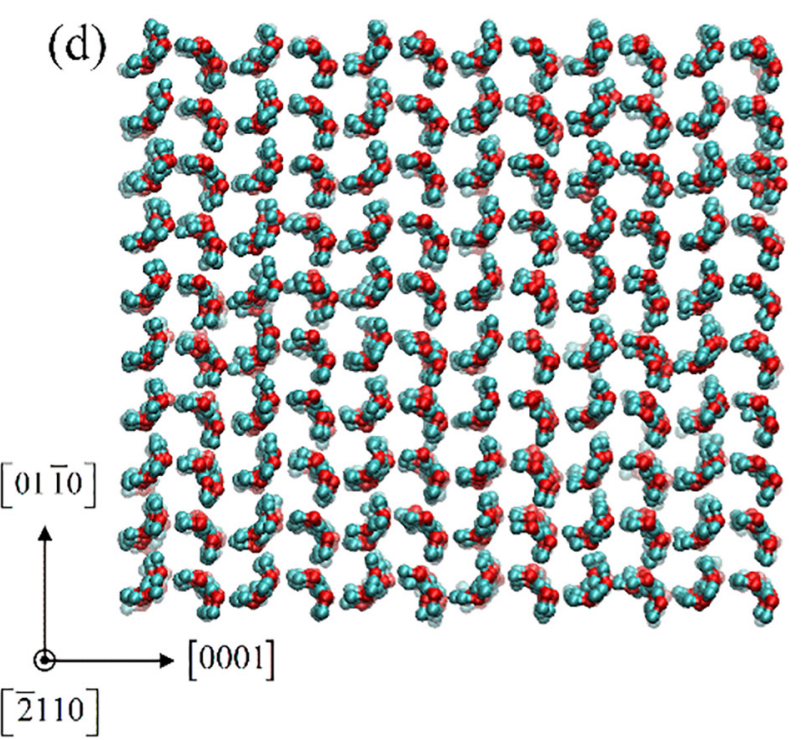


(a)

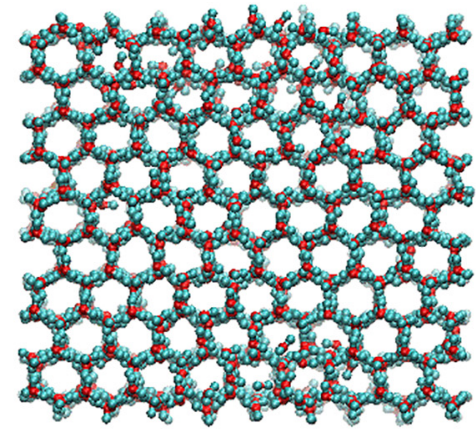

$6.0 \%$

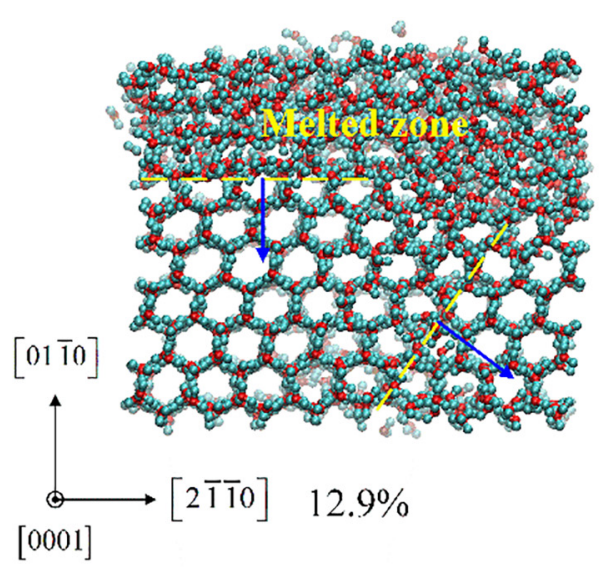

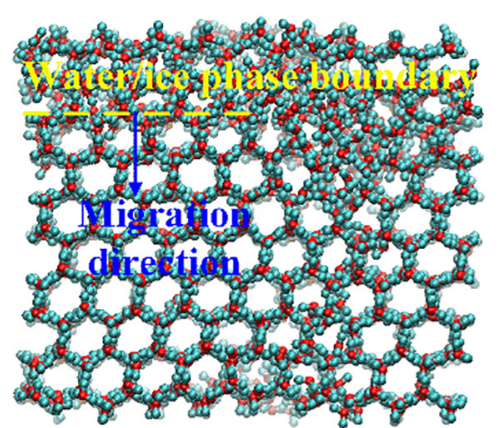

$9.0 \%$

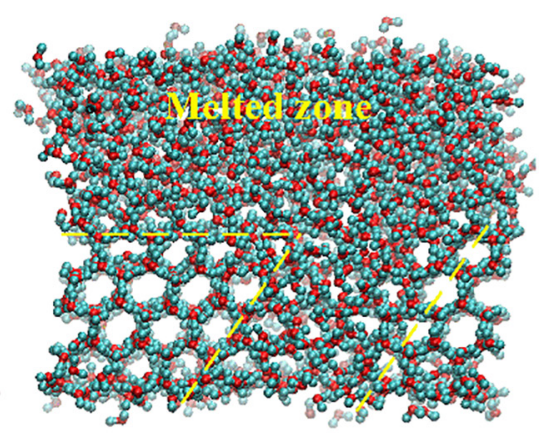

$18.0 \%$

Typical hexagonal cells in melted/unmelted area

(b)

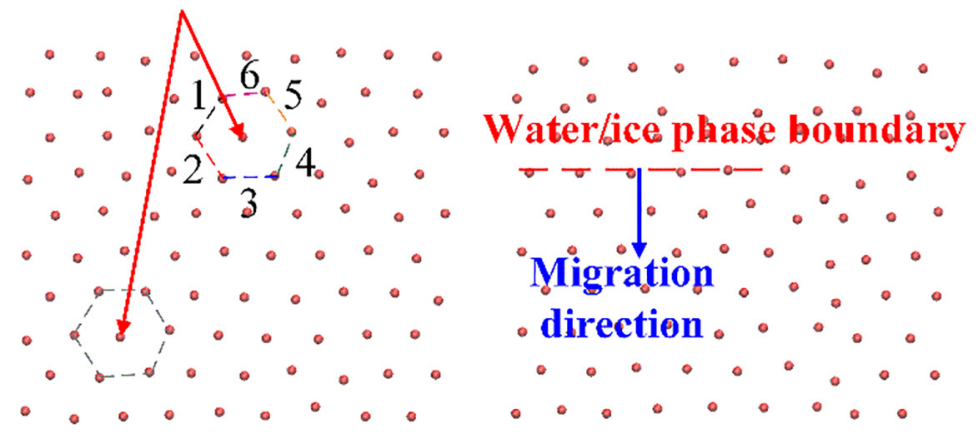

$6.0 \%$

$9.0 \%$

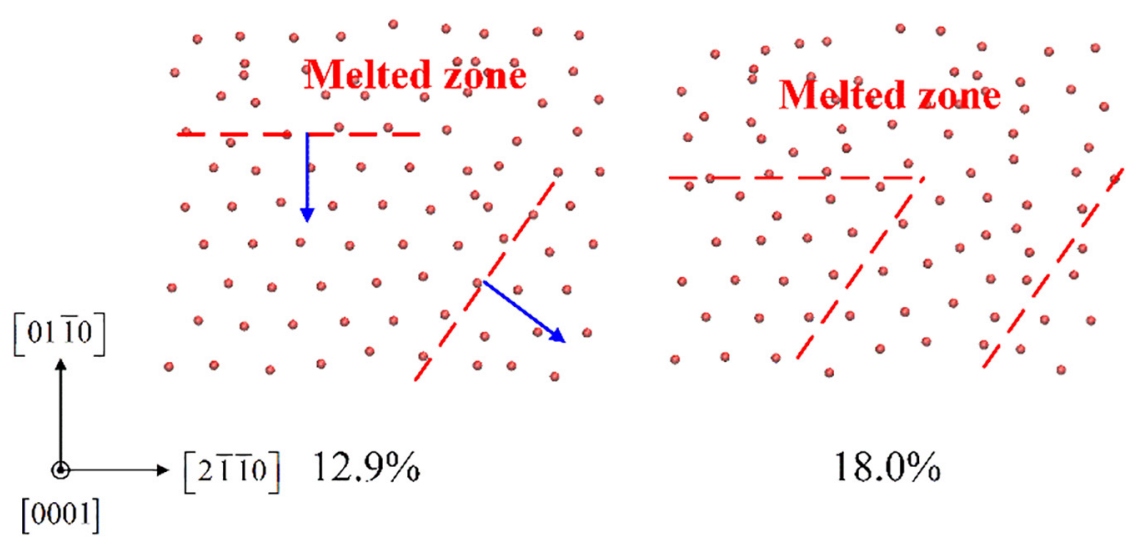

Fig. 3. Atomic structural evolution of ice at an initial temperature of $242 \mathrm{~K}$ from the perspective of (a) all of the atoms and (b) only one layer of oxygen atoms for clarity. 

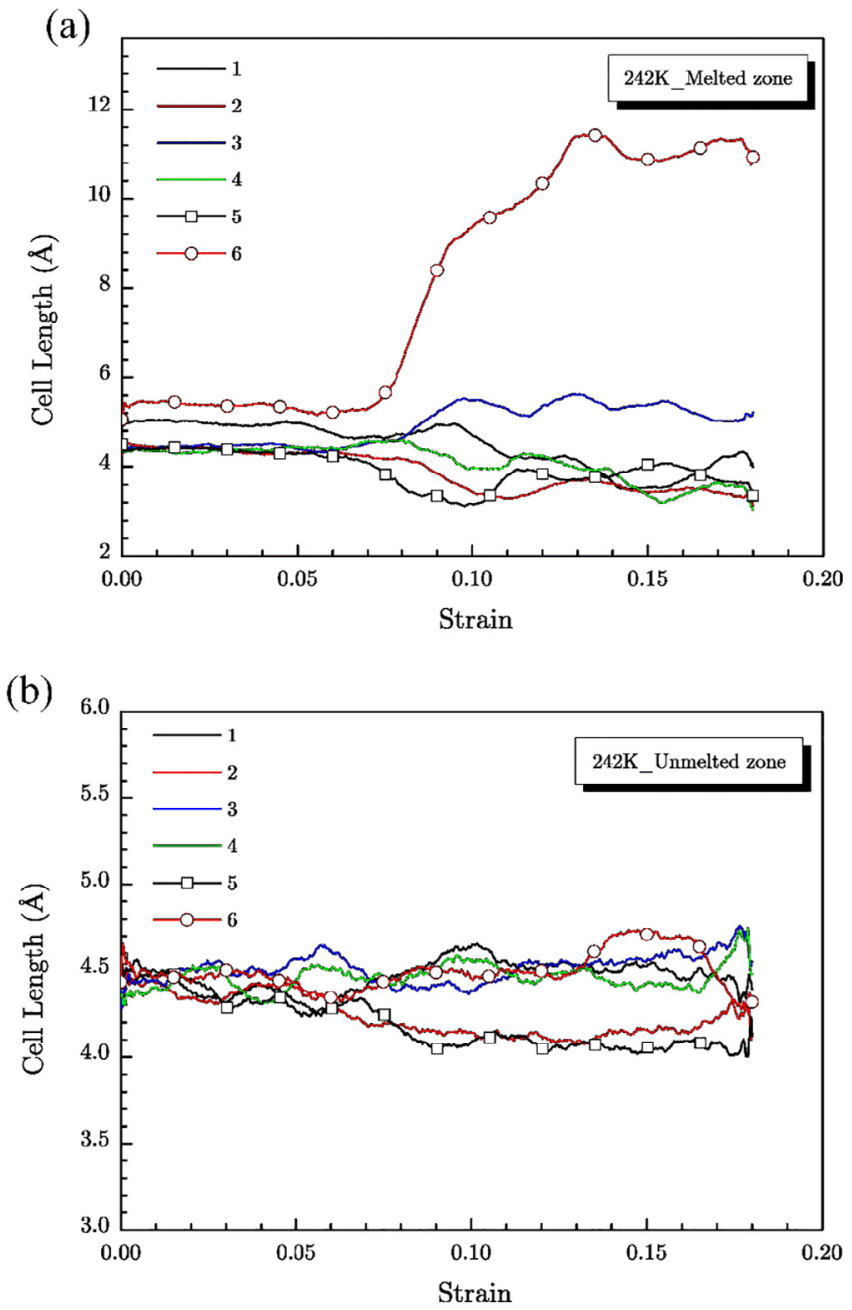

Fig. 4. The changes in the lengths of typical hexagonal cells of ice in (a) the melted area and (b) the unmelted area at an initial temperature of $242 \mathrm{~K}$.

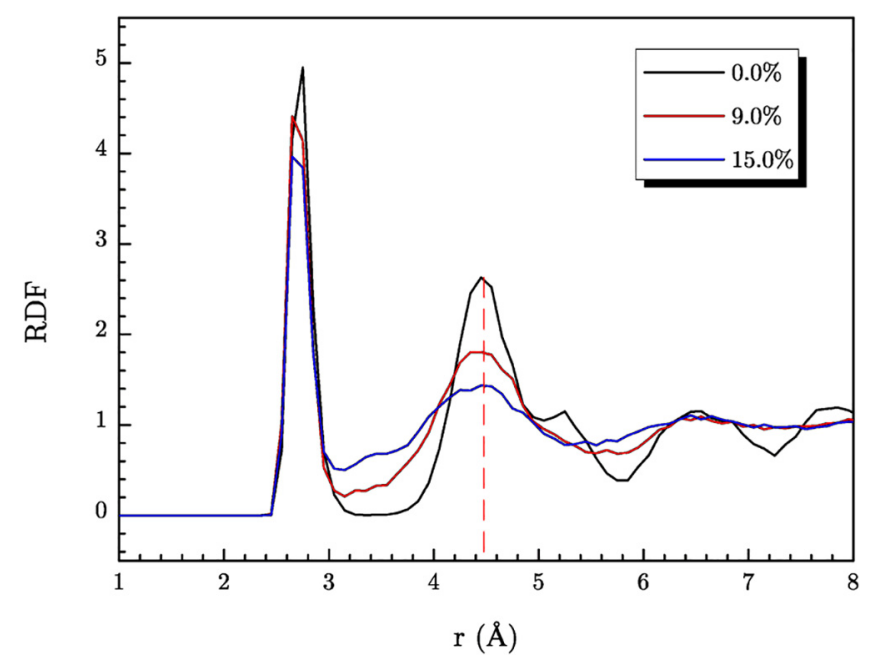

Fig. 5. RDF of the oxygen atoms at an initial temperature of $242 \mathrm{~K}$.

The entire simulation model with a dimension of $4.0 \mathrm{~nm} \times 3.9 \mathrm{~nm} \times 4.4 \mathrm{~nm}$ included 2160 water molecules. The period boundary condition applied on the three dimensions was employed to diminish the size effect. The initial ice structure is shown in Fig. 2(a)

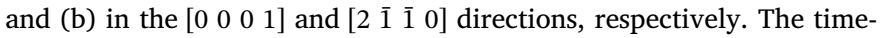
step was $1 \mathrm{fs}$. During thermo-relaxing, the Nose-Hoover method [34] was used to control the temperature and pressure. The initial temperature was $263 \mathrm{~K}$ and the pressure was 1 atmosphere. The entire relaxing time was approximate $300 \mathrm{ps}$, achieved by $3 \times 10^{5}$ steps, which was sufficiently long for the structure to attain thermal equilibrium. The structure of the ice after relaxing is shown in Fig. 2(c) and (d).

After relaxing, the system was cooled at $0.1 \mathrm{~K} / \mathrm{ps}$ to $242 \mathrm{~K}, 191 \mathrm{~K}$, and $163 \mathrm{~K}$, respectively. Then the systems were further relaxed for 100 ps before loading. The compressing loading that aligned with the armchair direction of the honeycomb structures, $\left[\begin{array}{llll}0 & \overline{1} & \overline{1} & 0\end{array}\right]$, was realised by controlling the displacement of the atoms with a strain rate of approximately $3 \times 10^{7} \mathrm{~s}^{-1}$ in $6 \mathrm{~ns}$ and $3 \times 10^{8} \mathrm{~s}^{-1}$ in $600 \mathrm{ps}$, respectively.

\section{Results and discussion}

\subsection{Temperature-dependent phase transformation}

The atomic structural evolution of ice, the changes in the lengths of a typical hexagonal cell of ice in the melting front, and the radial distribution function (RDF) [35] of oxygen atoms at an initial temperature of $242 \mathrm{~K}$ are shown in Figs. 3-5, respectively. As demonstrated in Fig. 3(a), melting occurred at a strain of approximately $6.0 \%$. The apparent solid-liquid interface formed as the strain increased to approximately $9.0 \%$. Afterward, the solid-liquid interface began to move towards the opposite side, that is, the $\left[\begin{array}{llll}0 & 1 & 1 & 0\end{array}\right]$ direction. Despite the gradual melting process, a shear melting process with a solid-liquid interface of $\left(\begin{array}{llll}1 & \overline{1} & 0 & 0\end{array}\right)$ occurred at a strain of $9.0 \%$. Then the newly formed solid-liquid interface migrated through the $\left[\begin{array}{llll}1 & \overline{1} & 0 & 0\end{array}\right]$ direction, leading to an expansion of the shear-induced melting zone. To better illustrate the structural evolution of ice, one slice layer of oxygen atoms on the (0001) crystal plane is shown in Fig. 3(b) (the hydrogen atoms were hidden for clarity). As demonstrated in Fig. 3(b), the hexagonal structures at the upper side of the system were damaged in response to the increase in the strain, and no regular structures were observed in this area, indicating the solid-liquid phase transformation.

To better understand the development of hexagonal cells, the changes in the lengths of one typical hexagonal cell of ice in the melting front area are provided in Fig. 4(a) and (b). The six lengths of each selected hexagonal cell were indexed counter-clockwise in No. 1-No. 6 as shown in Fig. 3(b) for clarity. The No. 6 cell length of the hexagonal cell in the melted region increased rapidly when the strain exceeded $6.8 \%$, indicating the local melting of ice. Before melting, the lengths of the hexagonal cell changed slightly due to the compression of the cell, that is, the decrease in the No. 2 and No. 5 cell lengths and the increase in the other four cell lengths indicated the elongation of the hexagonal cell along the $\left[\begin{array}{llll}1 & 0 & 1 & 0\end{array}\right]$ direction. In addition, the RDF of the oxygen atoms as shown in Fig. 5 demonstrated that the positions of the first and the second peak did not change, indicating no solid-solid phase transformation.

The atomic structural evolution of ice, the change in the lengths of a typical hexagonal cell of ice in the large deformation/untransformed area, and the RDF of the oxygen atoms at an initial temperature of $163 \mathrm{~K}$ are shown in Figs. 6-8, respectively. The deformation mechanism at $163 \mathrm{~K}$ was entirely different from that at $242 \mathrm{~K}$. As shown in Fig. 6(a), no melting phenomenon was observed in response to loading strain as high as $12.9 \%$. The initiation of the solid-solid phase transformation occurred once the strain reached $5.7 \%$. The newly formed solid phase was denoted by an ice-variant as depicted in Fig. 6(c), which demonstrates that the single honeycomb structure was squashed, and the newly formed structure was regarded as a monoclinic structure. The phase boundary between the parent ice- $1 \mathrm{~h}$ and the ice-variant was the ( $\left.\begin{array}{llll}1 & 0 & \overline{1} & 0\end{array}\right)$ crystal plane. The newly formed ice-variant band widened with the increase in the strain. In addition, dislocation-like glide phenomenon was observed when the strain reached approximately $10.5 \%$.

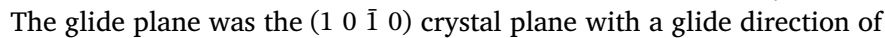
$\left[\begin{array}{llll}\overline{1} & 2 & \overline{1} & 0\end{array}\right]$. The dislocation-like glide initiated inside the ice-variant area 


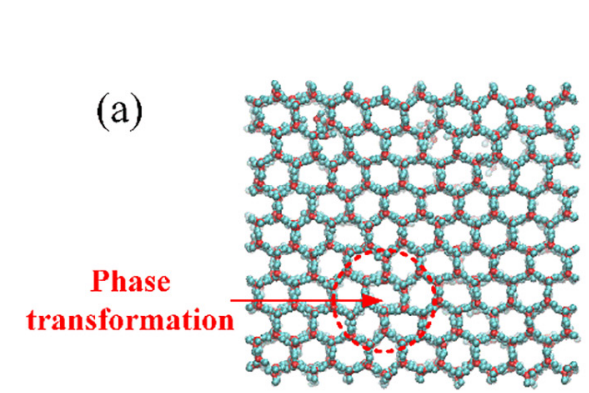

$5.7 \%$

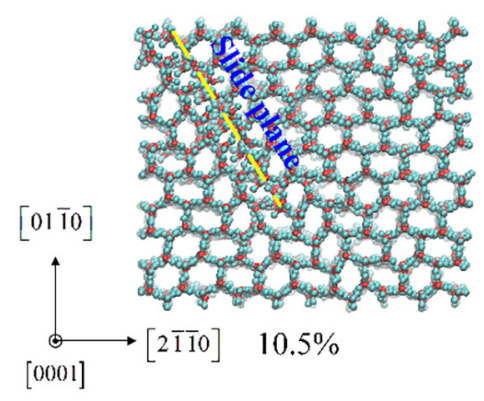

Typical hexagonal cells in large deformation

(b)

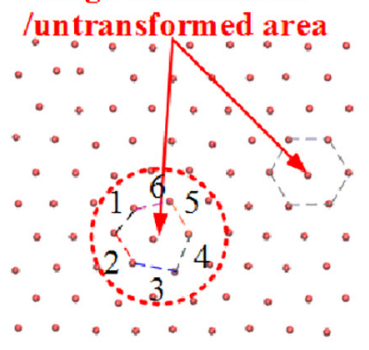

$5.7 \%$
$6.6 \%$

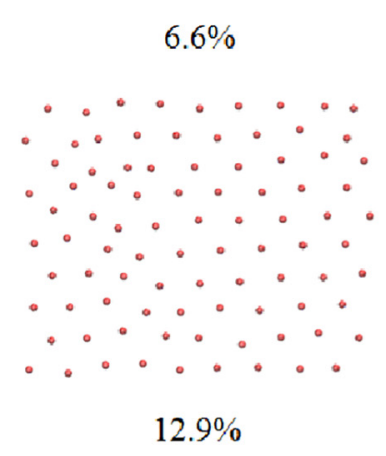

$12.9 \%$

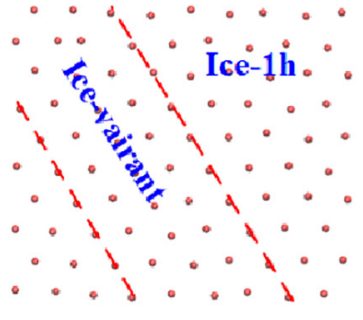

$6.6 \%$
"Dislocation-like" glide initiation

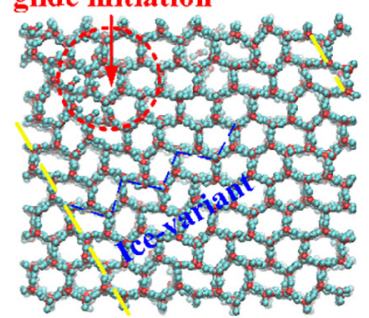

$9.0 \%$

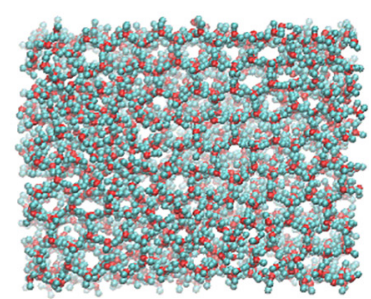

$18.0 \%$
$9.0 \%$

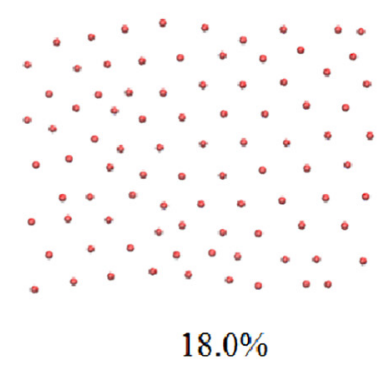

$18.0 \%$

(c)

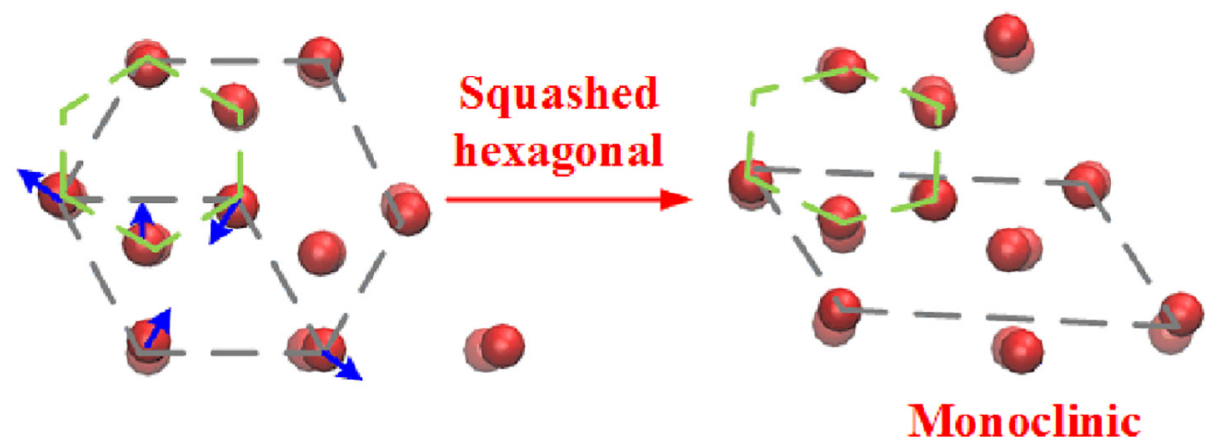

Fig. 6. Atomic structural evolution of ice from the perspective of (a) all of the atoms, (b) only one layer of oxygen atoms for clarity, and (c) the single crystal structure at an initial temperature of $163 \mathrm{~K}$. 


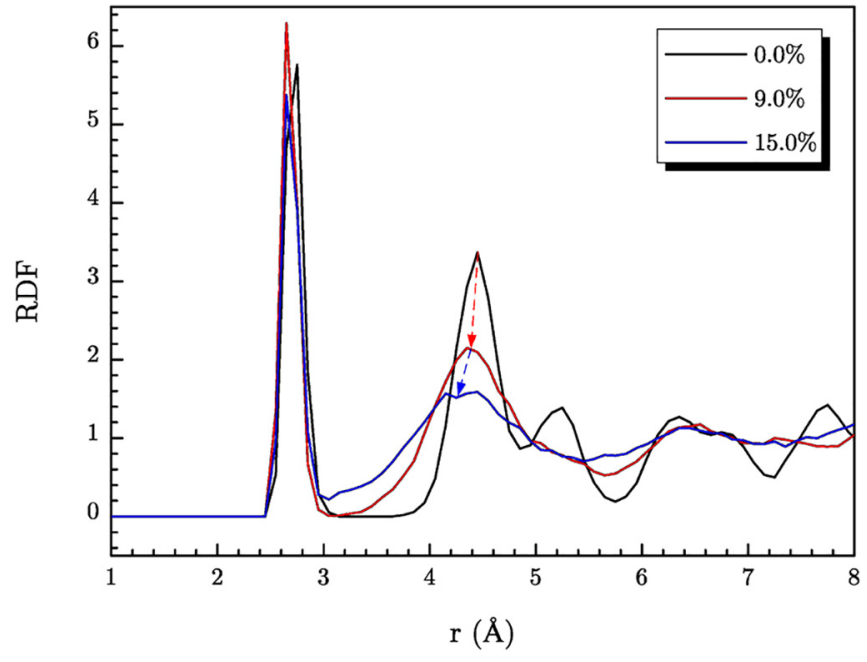

Fig. 7. RDF of the oxygen atoms at an initial temperature of $163 \mathrm{~K}$.
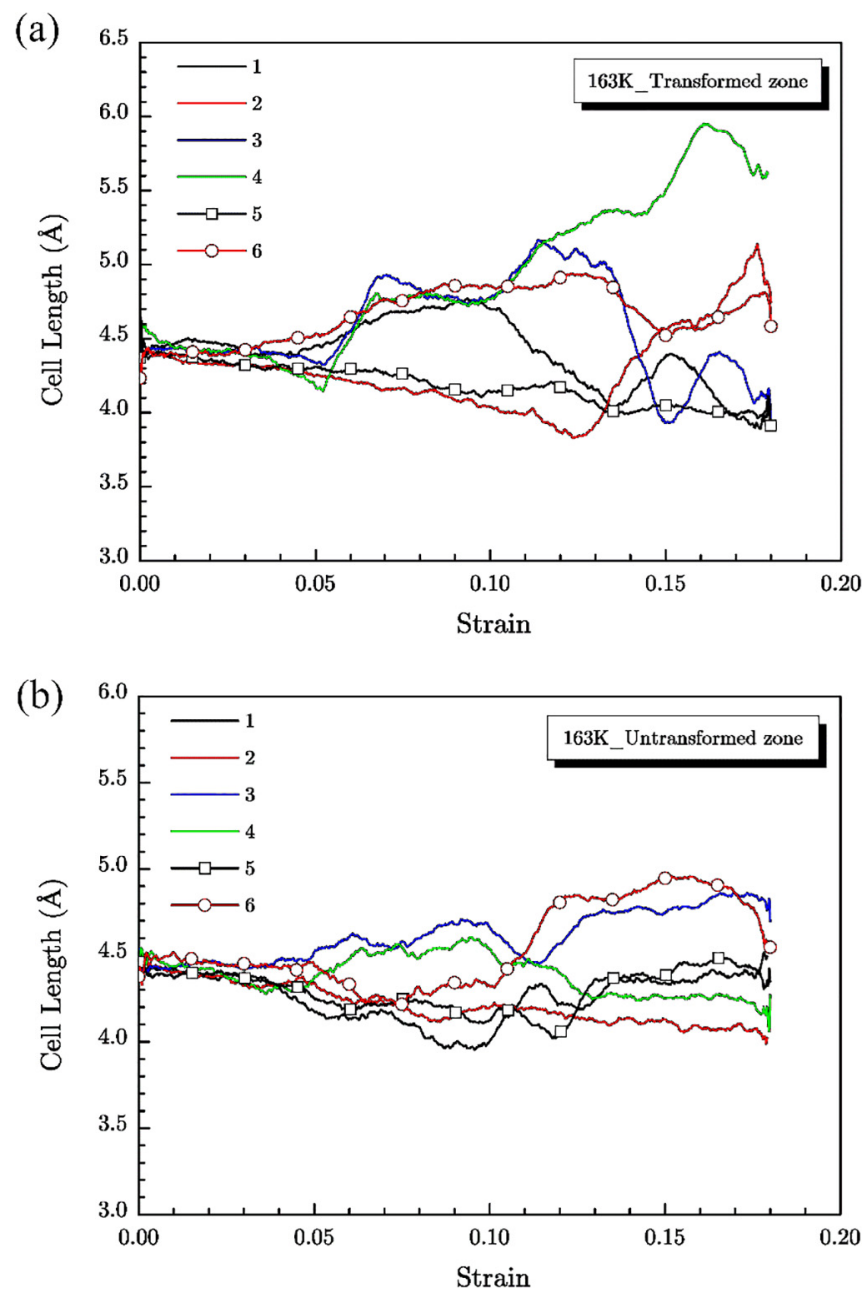

Fig. 8. The change in lengths of typical hexagonal cells of ice in (a) the transformed area and (b) the untransformed area at an initial temperature of $163 \mathrm{~K}$.

rather than at the boundary between the ice-variant and the ice- $1 \mathrm{~h}$.

One ice structure slice layer is shown in Fig. 6(b) (only the oxygen atoms are presented for clarity). As demonstrated in Fig. 6(b), the original orthohexagonal cell transformed into a hexagonal cell in the large deformation area as evidenced by the RDF evolution shown in Fig. 7, in which the position of the second peak continuously moved towards the first peak, indicating the structural transformation from ice- $1 \mathrm{~h}$ into an ice-variant.

Similarly, the typical change in the lengths of the hexagonal cells of the ice in the regions with and without phase transformation are demonstrated in Fig. 8(a) and (d), respectively. As shown in Fig. 8(a), after the strain exceeded $5.7 \%$, the No. 2 and No. 5 cell lengths decreased slightly while the other four lengths increased rapidly to plateaus, indicating the formation of a new solid state. With the further increase in the strain, the lengths of the newly formed hexagonal cell remained almost unchanged, implying the high stability of the newly formed structure. Afterward, the No. 4 cell length began to increase again at a strain of $13.0 \%$. However, the other five cell lengths were likely at a balance length of $4.5 \AA$. Moreover, the No. 4 cell length began to decrease to the balance length at a strain of $16.3 \%$, indicating the reorganisation of the hexagonal cell. However, as shown in Fig. 8(b), the No. 3 and No. 6 lengths of the hexagonal cell at the untransformed area increased slightly under loading while the other four lengths remained almost constant, indicating that the original orthohexagonal

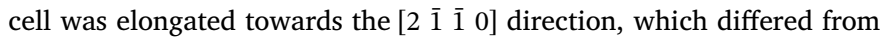
the results at a relatively high temperature of $242 \mathrm{~K}$.

\subsection{Temperature effects on the dynamic behaviour of ice}

The stress-strain relationship and the temperature evolution during compression at various initial temperatures are shown in Fig. 9. As demonstrated in Fig. 9(a), the strength of the ice increased with the decrease in the initial temperature, that is, the strength increased from $60.8 \mathrm{MPa}$ at $242 \mathrm{~K}$ to $121.7 \mathrm{MPa}$ at $163 \mathrm{~K}$, which was consistent with the experiment results obtained by $\mathrm{Wu}$ and Prakash [1]. At a relatively high temperature of $242 \mathrm{~K}$, the stress increased linearly in response to loading strain smaller than $6.0 \%$. Afterward, local melting started, and the stress increased slowly in response to further loading, which could be explained by the loading capacity of water with bulk compressibility under extremely high loading rates. However, once the initial temperature decreased, different stress-strain curves featuring piecewise linear stages due to the phase transformation from ice-1h to variant ice were observed, which could explain the experimental findings obtained by Wu and Prakash [1]. In addition, the stress dropped rapidly after the occurrence of the dislocation-like glide, different from that at relatively higher initial temperatures where the stress experienced no stress drop.

The temperature evolutions during compression at various initial temperatures are shown in Fig. 9(b). Due to the local melting, that is, a physical endothermic reaction, the system temperature began to decrease continuously with the increase in the strain up to $6.0 \%$. The temperature difference between the initial state and the final state was approximately $16 \mathrm{~K}$. However, when the initial temperature decreased to approximately $191 \mathrm{~K}$, the decrease in the temperature over the loading history decreased significantly to approximately $6 \mathrm{~K}$ compared to the higher temperature of $242 \mathrm{~K}$, which could be explained by the deformation behaviour of ice at various temperatures. At $191 \mathrm{~K}$, in addition to the ice- $1 \mathrm{~h}$ to the variant phase transformation, dislocationlike glide and local melting also occurred during compression. The competition between the endothermic solid-solid and solid-liquid phase transformation and the exothermic dislocation-like glide led to a drastic reduction in the decrease in the temperature over the loading process. In addition, when the temperature decreased to $163 \mathrm{~K}$, the local melting was not obvious during the simulation, implying that the temperature decrease at $163 \mathrm{~K}$ was solely induced by the phase transformation from ice- $1 \mathrm{~h}$ to the variant. The temperature increased slightly at the beginning of the loading process at $163 \mathrm{~K}$, followed by a rapid decrease in the temperature due to the predominant endothermic solid-solid phase transformation with the continuous increase in the strain to $10.5 \%$ and sequentially an equilibrium state at a temperature of $160 \mathrm{~K}$ because of the presence of the exothermic dislocation-like glide. Moreover, at the end of loading, the temperature slightly increased again, implying the 


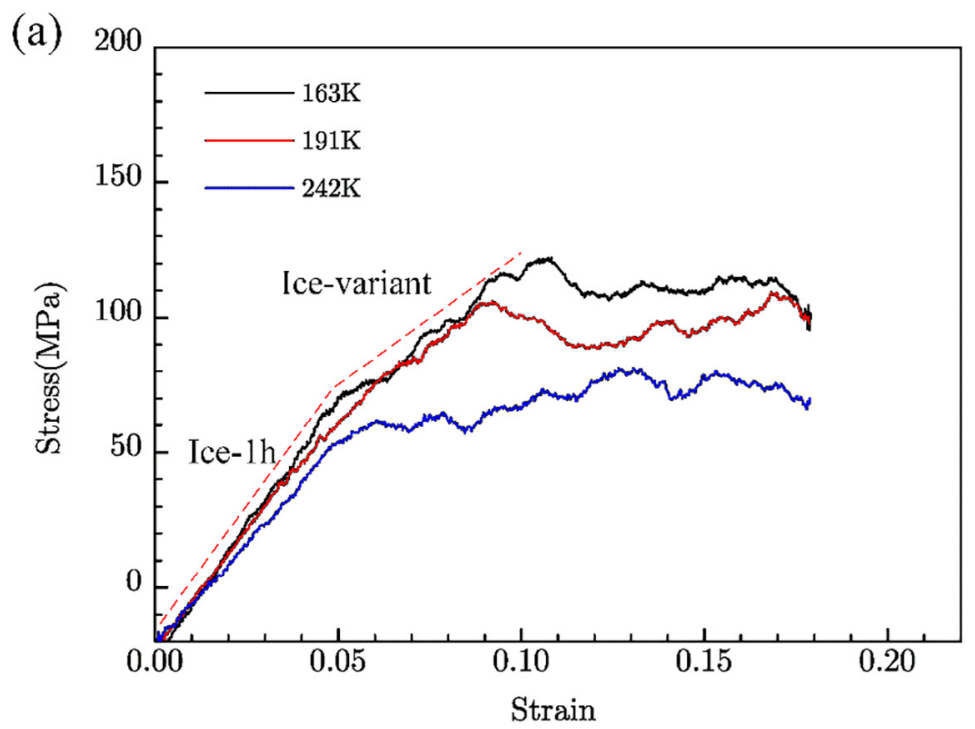

(b)
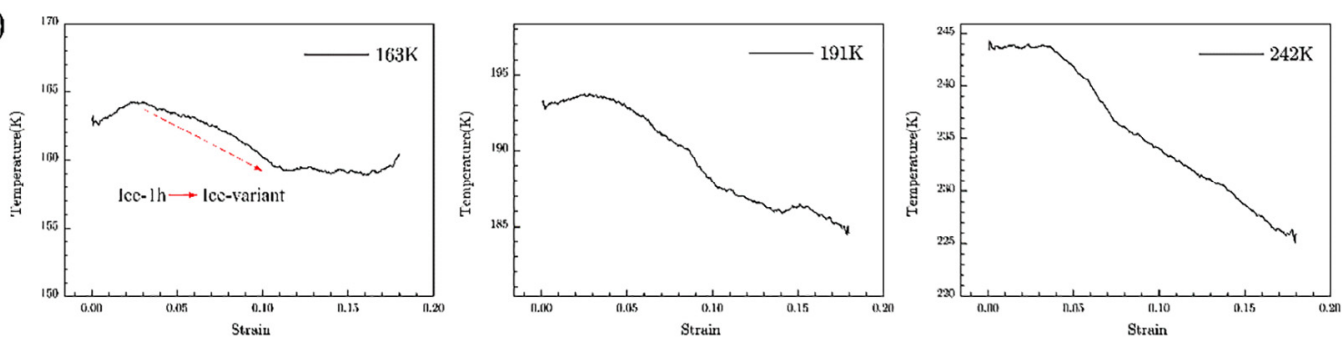

Fig. 9. (a) The stress-strain curves and (b) temperature evolution of the ice during compression at different initial temperatures.

predominance of the exothermic dislocation-like glide in this stage.

The solid-solid phase transformation mechanism obtained in the present study is helpful for understanding the anomalous phenomenon of the temperature-dependent strength of ice as observed by Wu and Prakash [1]. The present study suggests that at relatively high test temperatures, the solid-solid phase transformation stress was higher than the ice-1h strength, and the failure of the ice, therefore, was dominated by the ice-1h strength that increased in response to the decrease in the temperature. However, with the further decrease in the test temperatures, the increase in the solid-solid phase transformation stress was slower than the increase in the ice- $1 \mathrm{~h}$ strength. The solidsolid phase transformation occurred during the deformation after a critical low temperature, in which the ice strength might have been dominated by the competition of solid-solid phase transformation and fracture/melt and remained constant during phase transformation. Moreover, the strength of the newly formed phase may have been insensitive to the temperature, resulting in the insensitivity of the ice strength with the test temperatures during the entire compression process. Further simulations will be conducted to investigate the dynamic response of ice during shock wave propagation to better understand the relationship between the solid-solid phase transformation and the ice strength.

\subsection{Strain rate effects on the dynamic behaviour of ice}

The stress-strain relationships at strain rates of $3 \times 10^{7}$ and $3 \times 10^{8} \mathrm{~s}^{-1}$ are shown in Fig. 10. The discrepancy of the crystal structural evolution is also depicted. Fig. 10(a) demonstrates that the ice strength decreased with the decrease in the strain rate. However, at each strain rate, the typical characteristics of the stress-strain curves at different temperatures, that is, two-step piecewise linear stages at relatively lower temperatures while only one linear stage at higher temperatures and the dislocation-like glide induced stress drops at lower temperatures, were similar.

As shown in Fig. 10 (b), at $163 \mathrm{~K}$, both the solid-solid phase transformation and the dislocation-like glide were observed under the strain rates of $3 \times 10^{7} \mathrm{~s}^{-1}$ and $3 \times 10^{8} \mathrm{~s}^{-1}$, and the discrepancy in the structural evolution of the two different strain rates was not obvious, indicating that the strain rate effect was insensitive at this temperature range. However, at $242 \mathrm{~K}$, the melting pattern at different strain rates changed as shown in Fig. 10 (c). At the strain rate of $3 \times 10^{7} \mathrm{~s}^{-1}$, the melting initiated on both crystal planes of $\left(\begin{array}{llll}1 & 0 & \overline{1} & 0\end{array}\right)$ and ( $\left(\begin{array}{llll}1 & \overline{1} & 0 & 0\end{array}\right)$ instead of gradually melting with the solid-liquid interface perpendicular to the loading direction at the higher strain rate of $3 \times 10^{8} \mathrm{~s}^{-1}$. At the strain rate of $3 \times 10^{7} \mathrm{~s}^{-1}$, the upper melting boundary moved towards the direction of $\left[\begin{array}{llll}1 & \overline{2} & 1 & 0\end{array}\right]$, while the lower melting boundary propagated along the direction of $\left[\begin{array}{llll}1 & 1 & 2 & 0\end{array}\right]$, leading to the eventual junction of the melting regions at the middle of the simulation cell and the formation of a melt band with left and right boundaries expanding towards the $\left[\begin{array}{llll}1 & 0 & 1 & 0\end{array}\right]$ and $\left[\begin{array}{llll}1 & 1 & 0 & 0\end{array}\right]$ directions, respectively, in response to the additional loading.

\section{Summary}

The present research assessed the dynamic behaviour of single crystal ice- $1 \mathrm{~h}$ via a molecular dynamics simulation. The temperature and strain rate-dependent phase transformation behaviour under uniform compression was investigated. Two different phase transformation modes, that is, solid-liquid phase transformation at a relatively high initial temperature and phase transformation from ice- $1 \mathrm{~h}$ to an icevariant at a relatively low initial temperature, were observed, from which the observations of Wu and Prakash [1] could be reasonably interpreted. Moreover, the dislocation-like glide was observed at a relatively low initial temperature. There was a significant reduction in the temperature decrease over the loading history, ending with an equilibrium temperature of the system at a relatively low ambient 
(a)

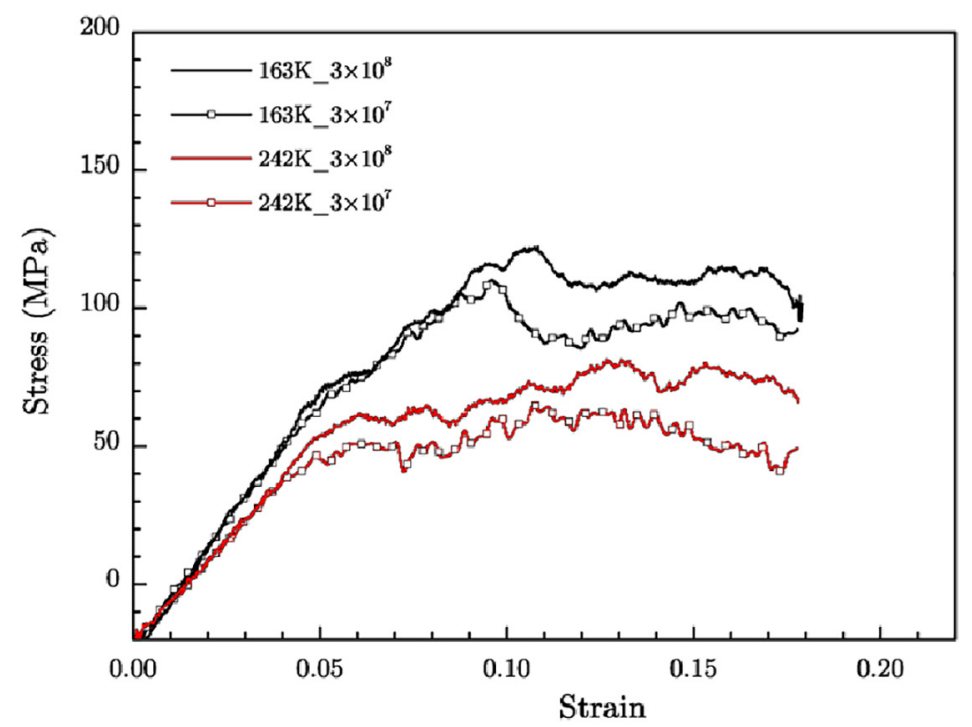

(b)

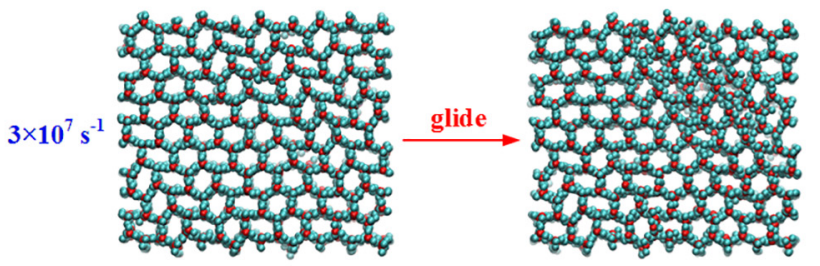

$5.7 \%$

$9.0 \%$

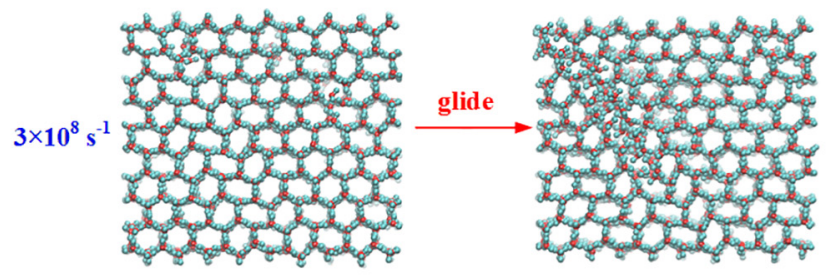

$5.7 \%$

$10.5 \%$

(c)

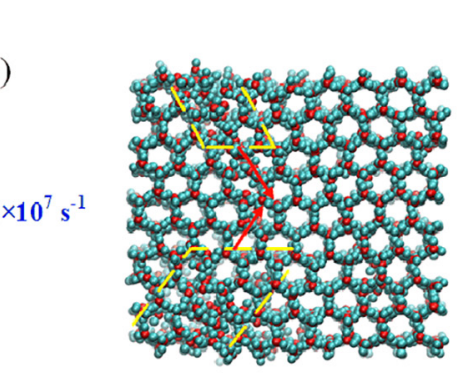

$5.0 \%$

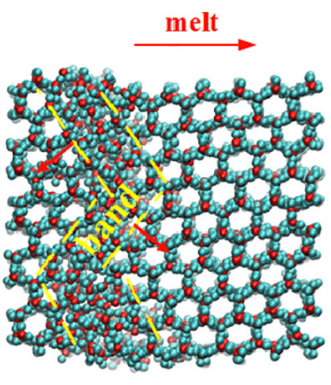

$7.0 \%$

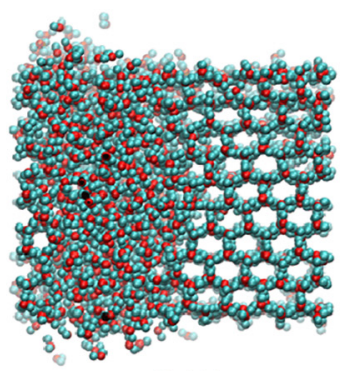

$13.2 \%$

$3 \times 10^{8} \mathrm{~s}^{-1}$

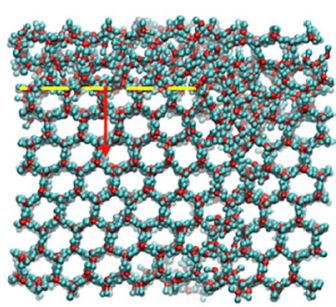

$9.0 \%$

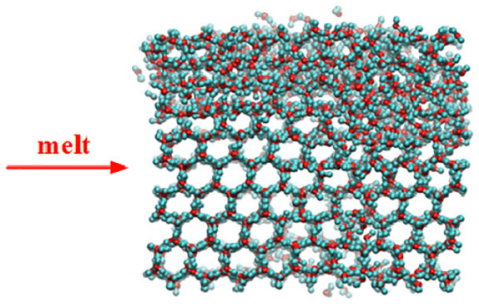

$12.9 \%$

Fig. 10. Effects of the strain rate and temperature on the (a) stress-strain curves, (b) structural evolution at $163 \mathrm{~K}$, and (c) structural evolution at $242 \mathrm{~K}$. 
temperature due to the competition between the endothermic solid-liquid phase transformation and the exothermic dislocation-like glide in the ice- $1 \mathrm{~h}$.

\section{CRediT authorship contribution statement}

Qiuyun Yin: Writing - original draft, Methodology, Funding acquisition. Lingling Hu: Data curation, Formal analysis. Xianqian Wu: Writing - review \& editing, Conceptualization, Funding acquisition. Kailu Xiao: Investigation, Validation. Chenguang Huang: Project administration, Formal analysis.

\section{Acknowledgements}

The authors appreciate the support of the National Natural Science Foundation of China under Grant Nos. 11802349, 11772363, and 11772347.

\section{Conflicts of interest}

Details on the potential conflicts of interest are in the publishing ethics section.

\section{References}

[1] X. Wu, V. Prakash, Cold Reg. Sci. Technol. 118 (2015) 1-13.

[2] R.M. Andrews, J. Glaciol. 31 (2017) 171-176.

[3] J.H. Currier, E.M. Schulson, Acta Metall. 30 (1982) 1511-1514.
[4] M. Mellor, Mechanical behavior of sea ice, in: N. Untersteiner (Ed.), The Geophysics of Sea Ice, Springer US, Boston, MA, 1986, pp. 165-281.

[5] E.M. Schulson, JOM 51 (1999) 21-27.

[6] E.M. Schulson, Eng. Fract. Mech. 68 (2001) 1839-1887.

[7] E.M. Schulson, D. Iliescu, 2005.

[8] E.M. Schulson, Acta Metall. Mater. 38 (1990) 1963-1976.

[9] L.J. Weber, W.A. Nixon, J. Offshore Mech. Arct. Eng. 118 (1996) 135-140.

[10] J.J. Petrovic, J. Mater. Sci. 38 (2003) 1-6.

[11] E.M. Schulson, E.T. Gratz, Acta Mater. 47 (1999) 745-755.

[12] A. Combescure, Y. Chuzel-Marmot, J. Fabis, Int. J. Solids Struct. 48 (2011) 2779-2790.

[13] M. Shazly, V. Prakash, B.A. Lerch, 2006.

[14] S.J. Jones, J. Phys. Chem. B 101 (1997) 6099-6101.

[15] H. Kim, J.N. Keune, J. Mater. Sci. 42 (2007) 2802.

[16] M. Shazly, V. Prakash, B.A. Lerch, Int. J. Solids Struct. 46 (2009) 1499-1515.

[17] X. Wu, V. Prakash, Int. J. Impact Eng. 76 (2015) 155-165.

[18] J. Lekner, Physica B 252 (1998) 149-159.

[19] O.A. Karim, A. Haymet, J. Chem. Phys. 89 (1988) 6889-6896.

[20] O.A. Karim, P.A. Kay, A. Haymet, J. Chem. Phys. 92 (1990) 4634-4635.

[21] M.A. Carignano, P.B. Shepson, I. Szleifer, Mol. Phys. 103 (2005) 2957-2967.

[22] V. Buch, P. Sandler, J. Sadlej, J. Phys. Chem. B 102 (1998) 8641-8653.

[23] K. Koga, G.T. Gao, H. Tanaka, X.C. Zeng, Nature 412 (2001) 802.

[24] G.T. Gao, X.C. Zeng, H. Tanaka, J. Chem. Phys. 112 (2000) 8534-8538.

[25] C. Vega, E. Sanz, J.L.F. Abascal, J. Chem. Phys. 122 (2005) 114507.

[26] J.A. Hayward, J.R. Reimers, J. Chem. Phys. 106 (1997) 1518-1529.

[27] X. Wei, S. Xiao, J. Ni, Mol. Simul. 36 (2010) 823-830.

[28] S.C. Gay, E.J. Smith, A.D.J. Haymet, J. Chem. Phys. 116 (2002) 8876-8880.

[29] M. Matsumoto, S. Saito, I. Ohmine, Nature 416 (2002) 409.

[30] S. Plimpton, J. Comput. Phys. 117 (1995) 1-19.

[31] P. Dalal, J. Knickelbein, A.D.J. Haymet, F.D. Sönnichsen, J.D. Madura, PhysChemComm 4 (2001) 32-36.

[32] K. Koga, X.C. Zeng, H. Tanaka, Phys. Rev. Lett. 79 (1997) 5262-5265.

[33] Z. Zhang, Z. Duan, Phys. Earth Planet. Inter. 149 (2005) 335-354.

[34] W.G. Hoover, Phys. Rev. A 31 (1985) 1695-1697.

[35] J. Barker, D. Henderson, Mol. Phys. 21 (1971) 187-191. 